\title{
Adult-onset Still's Disease Associated with Scrub Typhus
}

\author{
Mi-Hye KWON, Chung-Il JOUNG \\ Department of Internal Medicine, Konyang University Hospital, Daejeon, South Korea
}

Adult-onset Still's disease (AOSD) is a rare autoinflammatory disorder with typical features of spiking fever, evanescent rash, and arthritis/ arthralgia. Pathogenesis of AOSD is not well known, and it is hypothesized that environmental triggers may initiate disease in individuals with genetic susceptibility. Anecdotal reports have indicated close temporal relationship between AOSD and various infections, which may suggest infectious triggers for developing AOSD, but rickettsial diseases were rarely reported..$^{1-5}$ A 51-year-old female patient presented with fever for one month. Three weeks ago, she was diagnosed as scrub typhus with fever, severe myalgia, arthralgia, rash, and eschar in the right arm at another hospital (Figure 1). She was a farmer living in Chungcheong province, a rural area of Korea. After introduction of oral doxycycline, patient felt better subjectively, but fever was relapsed. She was treated with empirical intravenous antibiotics for fever of unknown origin for 10 days. She began to complain of pain in the wrists, knees, and ankles and was transferred to our hospital. Spiking fever of $38.3^{\circ}$ Celcius once or twice a day was noted. There was maculopapular rash on upper chest and both lower legs, which was minimally itchy and not evanescent. Painful joints were tender but not swollen. Laboratory findings were as follows; white blood cell: $13,700 / \mu \mathrm{L}$, hemoglobin: $9.9 \mathrm{~g} / \mathrm{dL}$, erythrocyte sedimentation rate: $83 \mathrm{~mm} / \mathrm{hr}, \mathrm{C}$-reactive protein: $10.3 \mathrm{mg} / \mathrm{dL}$, aspartate aminotransferase: $124 \mathrm{IU} / \mathrm{L}$, alanine aminotransferase: 131 IU/L, ferritin $>2000 \mathrm{ng} / \mathrm{mL}$ (4.63-204.0), rheumatoid factor $6.9 \mathrm{IU} / \mathrm{mL}$, antinuclear antibody 1:80. Antibodies for viral and rickettsial diseases were negative. Abdominal computed tomography revealed several enlarged lymph nodes around common hepatic artery and hepatoduodenal ligament, and other results for evaluation of fever of unknown origin were negative. Patient had severe sore throat during admission and we diagnosed her as having AOSD by Yamaguchi criteria.

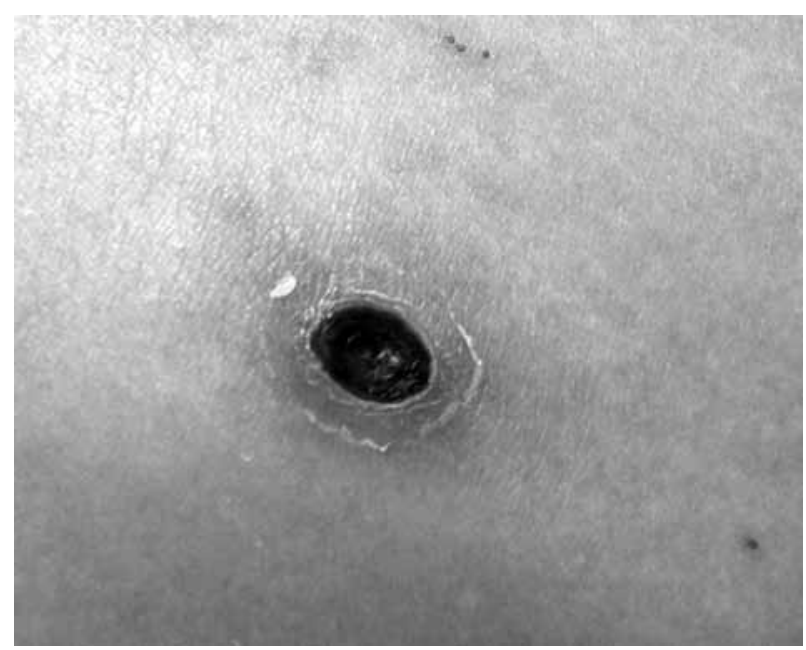

Figure 1. Typical inoculation eschar on right arm. Black necrotic center with surrounding erythematous scaly lesion is observed.

Received: March 26, 2015 Accepted: March 28, 2015 Published online: June 08, 2015

Correspondence: Chung-Il Joung, MD. Department of Internal Medicine, Konyang University Hospital, 302-718 Daejeon, South Korea.

Tel: +81 1030763029 e-mail: cij1221@kyuh.ac.kr

(2015 Turkish League Against Rheumatism. All rights reserved. 
She was treated with high dose prednisolone and oral weekly methotrexate. There was dramatic clinical and laboratory improvement, and she was discharged. As AOSD and infections have apparent similarities such as acute onset, high fever, generalized lymphadenopathy, and elevated inflammatory markers, infectious triggers have been suggested for the cause of AOSD. The incriminated infectious agents reported to date are rubella, Echovirus 7, mumps, cytomegalovirus, parainfluenza, coxsackievirus, adenovirus, influenza $A$, human herpes virus 6, hepatitis B, parvovirus B19, Epstein-Barr virus, Yersinia enterocolitica, Chlamydophila pneumoniae, Mycoplasma pneumoniae, Brucella abortus, and Borrelia burgdorferi. There were close temporal relationships between infections and following AOSD, suggesting infectious triggers. To develop AOSD, infectious triggers and immunological processes may interact in individuals with genetic susceptibilities. In our patient, close temporal relationship between scrub typhus and AOSD strongly suggested that scrub typhus triggered AOSD. Scrub typhus, a vector born rickettsial disease caused by Orientia tsutsugamushi, is endemic in Eastern Asia including South Korea. Although symptoms of the two diseases are substantially similar, scrub typhus is readily treatable with antibiotics in majority of patients. Our patient had initial response to doxycycline but later developed spiking fever and arthralgia. The improvement with steroid therapy indicated that her later illness was not caused by scrub typhus. In this article, we report a case of AOSD following scrub typhus and suggest that scrub typhus may be a trigger for the development of AOSD.

\section{Declaration of conflicting interests}

The authors declared no conflicts of interest with respect to the authorship and/or publication of this article.

\section{Funding}

The authors received no financial support for the research and/or authorship of this article.

\section{REFERENCES}

1. Balleari E, Cutolo M, Accardo S. Adult-onset Still's disease associated to toxoplasma gondii infection. Clin Rheumatol 1991;10:326-7.

2. Bento DP, Tavares R, Baptista Leite R, Miranda A, Ramos S, Ventura F, et al. Adult-Onset Still's Disease and cytomegalovirus infection. Acta Reumatol Port 2010;35:259-63.

3. Seo SR, Kim SS, Lee SJ, Kim TJ, Park YW, Lee SS. Adult-onset Still disease in a patient with acute hepatitis A. J Clin Rheumatol 2011;17:444-5.

4. Yoo WH. Adult onset Still's disease following influenza vaccination. Joint Bone Spine 2010;77:373-4.

5. Perez C, Artola V. Adult Still's disease associated with Mycoplasma pneumoniae infection. Clin Infect Dis 2001;32:105-6. 